\title{
Renormalization of the neutrino mass matrix
}

\author{
T. K. Kuo ${ }^{1}$, James Pantaleone ${ }^{2}$, Guo-Hong $\mathrm{Wu}^{3}$ \\ ${ }^{1}$ Department of Physics, Purdue University, West Lafayette, IN 47907* \\ ${ }^{2}$ Department of Physics, University of Alaska, Anchorage, Alaska 99508, \\ ${ }^{3}$ Institute of Theoretical Science, University of Oregon, Eugene, OR 97403
}

(Revised, August 2001)

\begin{abstract}
The renormalization group equations for the general $2 \times 2$, complex, neutrino mass matrix are shown to have exact, analytic solutions. Simple formulas are given for the physical mixing angle, complex phase and mass ratio in terms of their initial values and the energy scales. We also establish a (complex) renormalization invariant relating these parameters. The qualitative features of the physical parameters' renormalization are clearly illustrated in vector field plots. In both the SM and MSSM, maximal mixing is a saddle point.
\end{abstract}

\footnotetext{
*Email: tkkuo@physics.purdue.edu, jim@neutrino.phys.uaa.alaska.edu, gwu@darkwing.uoregon.edu
} 


\section{Introduction}

Recent experiments [1], 2] have revealed some salient features of the neutrino mass matrix. It is now rather well-established that the neutrino masses are tiny, and that at least some of the mixing angles are large, or even maximal. Considerable efforts have been devoted to a theoretical understanding of these features. Any theoretical model faces an important issue. Is the model valid at the high or low energy scale? How are the physical parameters related between the two scales? These questions are answered by studying the renormalization group equation (RGE). In addition, RGE may offer a natural mechanism which can drive the neutrino mixing angle to its maximal value.

The RGEs governing the neutrino mass matrix were worked out some time ago [3] and have been studied extensively (see e.g. [4, 5]). Most studies considered the case of real matrices, although some dealt with complex ones also. In this paper, we wish to point out that there is an exact, analytic solution for the RGE of the general $2 \times 2$ mass matrix. Simple formulas are given for the physical mixing angle, complex phase and mass ratio in terms of their initial values at an energy scale. We further show that there is a (complex) RGE invariant relating the running of these three variables. The fixed points of the physical parameters are obtained and their stability is determined. The nature of the running near these fixed points is clearly illustrated in vector field plots [6].

In both the standard model (SM) and the minimal supersymmetric standard model (MSSM), the effective Majorana neutrino mass matrix can arise from a dimension-five operator. Its RGE (in the basis where the charged-lepton mass matrix is diagonal) is given by $\left(t=\frac{1}{16 \pi^{2}} \ln \mu / M_{X}\right)$,

$$
\frac{d}{d t} m_{\nu}=-\left(\kappa m_{\nu}+m_{\nu} P+P^{T} m_{\nu}\right)
$$

where $\kappa$ depends on a combination of the coupling constants and, for two flavors (for definiteness, we consider $\nu_{\mu}$ and $\nu_{\tau}$ ) [3], after absorbing the muon Yukawa coupling term into $\kappa$,

$$
\begin{gathered}
P=P^{T}=\chi\left(1-\sigma_{3}\right), \\
\chi=\left\{\begin{array}{cl}
\left(y_{\tau}^{2}-y_{\mu}^{2}\right) / 4, & \text { SM } \\
-\left(\tilde{y}_{\tau}^{2}-\tilde{y}_{\mu}^{2}\right) / 2, & \text { MSSM }
\end{array}\right.
\end{gathered}
$$

Here $y_{\tau}=\sqrt{2} m_{\tau} / v$ and $\tilde{y}_{\tau}=\sqrt{2} m_{\tau} /(v \cos \beta)$ are the $\tau$ Yukawa couplings in the SM and MSSM respectively, with $v \simeq 246 \mathrm{GEV}$ and $\tan \beta$ is given by the ratio of the two Higgs VEVs in MSSM. Also, $y_{\mu}$ and $\tilde{y}_{\mu}$ are similarly defined.

\section{Solution}

A formal solution [5] to Eq. (11) is

$$
m_{\nu}(t)=e^{-\kappa^{\prime} t} e^{\xi \sigma_{3}} m_{\nu}(0) e^{\xi \sigma_{3}}
$$


where we have ignored the $t$-dependence of the coupling constants so that $\int \kappa d t \simeq \kappa t$, etc., and

$$
\begin{aligned}
\kappa^{\prime} & =\kappa+2 \chi \\
\xi & =\chi t .
\end{aligned}
$$

It is convenient to factor out the determinant

$$
m_{\nu}=\sqrt{m_{1} m_{2}} M
$$

then $\operatorname{det} M=+1$, and the mixing angle and the complex mass ratio are contained solely in $M$. We have

$$
\begin{aligned}
\sqrt{m_{1}(t) m_{2}(t)} & =e^{-\kappa^{\prime} t} \sqrt{m_{1}(0) m_{2}(0)} \\
M(t) & =e^{\xi \sigma_{3}} M(0) e^{\xi \sigma_{3}}
\end{aligned}
$$

The overall scale $\sqrt{m_{1} m_{2}}$ has a simple dependence on $t$. The mixing angle and the (complex) mass ratio evolve via a transformation that is similar to the effects of the familiar seesaw model [7]. The difference lies only in that while $|\xi| \gg 1$ for the seesaw model, for the RGE usually $|\xi| \ll 1\left(\sim 10^{-5}\right.$ for the SM). Nevertheless, the general analytic solution obtained earlier [8, 9] is equally valid here. We will now discuss this solution in the context of RGE.

As was shown earlier [9], a symmetric and complex $2 \times 2$ matrix with det $=1$ can be parametrized in a standard way. We write

$$
\begin{aligned}
M(t) & =U(t) e^{-2 \eta \sigma_{3}} U(t)^{T} \\
U(t) & =e^{-i \alpha \sigma_{3}} e^{-i \theta \sigma_{2}} e^{-i \phi \sigma_{3}}
\end{aligned}
$$

where $\eta$ is given in terms of the mass eigenvalues by

$$
\eta=\frac{1}{4} \ln \frac{m_{2}}{m_{1}} .
$$

At $t=0, M(0)$ and $U(0)$ are similarly defined in terms of $\left(\eta_{0}, \theta_{0}, \alpha_{0}, \phi_{0}\right)$. Here $m_{2}$ and $m_{1}$ are, by definition, positive definite. The relative phase of the mass eigenvalues is given by $4 \phi$ so that $M$ becomes real for $\phi=0$ and $\phi=\pi / 4$, corresponding to the same sign and opposite sign mass values, respectively; $\theta$ is the physical mixing angle. The phase $\alpha$ can be absorbed into the arbitrary phase of the charged leptons and is not observable. However, as emphasized before (see refs. [4] and [9]), it evolves with t and so influences the evolution of the other parameters. We can rewrite $M=M(t)$ as [9]

$$
M=\left(\begin{array}{cc}
e^{-2 i \alpha}\left(\operatorname{ch} 2 \bar{\eta}-c_{2 \theta} \operatorname{sh} 2 \bar{\eta}\right) & -s_{2 \theta} \operatorname{sh} 2 \bar{\eta} \\
-s_{2 \theta} \operatorname{sh} 2 \bar{\eta} & e^{2 i \alpha}\left(\operatorname{ch} 2 \bar{\eta}+c_{2 \theta} \operatorname{sh} 2 \bar{\eta}\right)
\end{array}\right)
$$

\footnotetext{
${ }^{1}$ To include the $t$-dependence, one needs only to replace $\kappa t$ by $\int \kappa d t$ etc, in the appropriate formulae in the following. Numerically, we have verified that the difference is negligible.
} 
where we use the notation $\operatorname{ch} 2 \bar{\eta}=\cosh 2 \bar{\eta}, c_{2 \theta}=\cos 2 \theta$, etc. and $\bar{\eta}=\eta+i \phi$.

The RGE evolution of the parameters is given by $M(0) \rightarrow M(t)$. From Eq. (9), it is obvious that the off-diagonal elements of $M$ do not evolve. We have thus the (complex) RGE invariant:

$$
s_{2 \theta} \operatorname{sh} 2 \bar{\eta}=s_{2 \theta_{0}} \operatorname{sh} 2 \bar{\eta}_{0}
$$

where the subscript 0 denotes the values at the high energy scale $t=0$. We note that in terms of the physical neutrino masses, $\operatorname{sh} 2 \bar{\eta}=\left(\sqrt{m_{2} / m_{1}} e^{i 2 \phi}-\sqrt{m_{1} / m_{2}} e^{-i 2 \phi}\right) / 2$.

Eq. (9) also implies two complex relations between the diagonal elements of $M(t)$ and $M(0)$. They can be used to solve for the four unknowns $(\alpha, \theta, \phi, \eta)$ in terms of their initial values $\left(\alpha_{0}, \theta_{0}, \phi_{0}, \eta_{0}\right)$. After a short calculation we find

$$
\begin{aligned}
\tan 2 \theta & =\frac{s_{2 \theta_{0}} /\left(c_{2 \Delta \alpha} \operatorname{ch} 2 \xi\right)}{c_{2 \theta_{0}}-\Sigma_{R} \tanh 2 \xi+\Sigma_{I} \tan 2 \Delta \alpha} \\
\tan 2 \Delta \alpha & =\frac{\Sigma_{I}}{c_{2 \theta_{0}}-\Sigma_{R} \operatorname{coth} 2 \xi} \\
\operatorname{coth} 2 \bar{\eta}_{0} & =\Sigma_{R}+i \Sigma_{I}=\frac{1-\left(m_{1} / m_{2}\right)_{0}^{2}-2 i\left(m_{1} / m_{2}\right)_{0} s_{4 \phi_{0}}}{1+\left(m_{1} / m_{2}\right)_{0}^{2}-2\left(m_{1} / m_{2}\right)_{0} c_{4 \phi_{0}}}
\end{aligned}
$$

where $\Delta \alpha=\alpha-\alpha_{0}$, the $\Sigma$ 's are real, and the masses in the last equation are at $t=0$. These solutions agree with Ref. [8], where they were obtained by a somewhat different method. We do not write down the solution for $\bar{\eta}$, since, knowing $\theta, \eta$ and $\phi$ can be determined by Eq. (14).

\section{RGE}

The RGEs for the physical parameters can be worked out. From Eq. (9), the RGE for $M(t)$ is

$$
\frac{d}{d t} M=\chi\left\{M, \sigma_{3}\right\}
$$

We may decompose $M$ into Pauli matrices,

$$
M=\Sigma_{i=0}^{3} M_{i} \sigma_{i},
$$

where the coefficients $M_{i}$ are complex, and $M_{2}=0$ since $M$ is symmetric. Then

$$
\begin{aligned}
\frac{d}{d t} M_{1} & =0 \\
\frac{d}{d t}\left(M_{0} \pm M_{3}\right) & = \pm 2 \chi\left(M_{0} \pm M_{3}\right) .
\end{aligned}
$$

Eq. (20), which says that the off-diagonal elements of $M$ do not run, is just another form of Eq. (14),

$$
\frac{d}{d t}\left(s_{2 \theta} \operatorname{sh} 2 \bar{\eta}\right)=0
$$


The diagonal elements of $M$ run according to

$$
\begin{aligned}
\frac{d}{d t}\left[e^{-2 i \alpha}\left(\operatorname{ch} 2 \bar{\eta}-c_{2 \theta} \operatorname{sh} 2 \bar{\eta}\right)\right] & =+2 \chi e^{-2 i \alpha}\left(\operatorname{ch} 2 \bar{\eta}-c_{2 \theta} \operatorname{sh} 2 \bar{\eta}\right) \\
\frac{d}{d t}\left[e^{2 i \alpha}\left(\operatorname{ch} 2 \bar{\eta}+c_{2 \theta} \operatorname{sh} 2 \bar{\eta}\right)\right] & =-2 \chi e^{2 i \alpha}\left(\operatorname{ch} 2 \bar{\eta}+c_{2 \theta} \operatorname{sh} 2 \bar{\eta}\right) .
\end{aligned}
$$

Eqs. (23) and (24) can be readily rewritten in terms of RGE for the various parameters. It is found that, for the unobservable phase $\alpha$,

$$
\frac{d}{d t} \alpha=\chi \frac{s_{4 \phi}}{\operatorname{sh} 4 \eta}
$$

The RGEs for the physical parameters are

$$
\begin{aligned}
& \frac{d \eta}{d t}=-\chi c_{2 \theta} \\
& \frac{d \phi}{d t}=-\chi c_{2 \theta} \frac{s_{4 \phi}}{\operatorname{sh} 4 \eta} \\
& \frac{d \theta}{d t}=\chi s_{2 \theta}\left[c_{2 \phi}^{2} \operatorname{coth} 2 \eta+s_{2 \phi}^{2} \tanh 2 \eta\right] .
\end{aligned}
$$

It is easy to verify that Eq. (22) is satisfied. Also, the last equation agrees with the known result for the case of real mass matrix [3, 4, 5]

$$
\frac{d \theta}{d t}=\chi s_{2 \theta} \frac{m_{2}+m_{1}}{m_{2}-m_{1}}
$$

valid for same $\operatorname{sign}(\phi=0)$ and opposite $\operatorname{sign}\left(\phi=\pi / 4\right.$, or $\left.m_{1} \rightarrow-m_{1}\right)$ mass values. Our result, with the convention of positive definite $m_{1}$ and $m_{2}$, interpolates these two equations. Note also that the equations can be expressed in terms of tanh $2 \bar{\eta}$, with $\left[c_{2 \phi}^{2} \operatorname{coth} 2 \eta+s_{2 \phi}^{2} \tanh 2 \eta\right]=1 / \operatorname{Re}(\tanh 2 \bar{\eta})$ and $s_{4 \phi} / \operatorname{sh} 4 \eta=\operatorname{Im}(\tanh 2 \bar{\eta}) / \operatorname{Re}(\tanh 2 \bar{\eta}) . \mathrm{Fi}-$ nally, the right-hand sides of Eqs. (25-28) are independent of the unobservable phase $\alpha$, as it should be. Explicitly, from Eq. (9), $e^{\xi \sigma_{3}}$ commutes with $e^{i \alpha \sigma_{3}}$, so that any RGE evolution does not depend on $\alpha$.

It is interesting to note that, for $\phi=0$, there is a simple geometrical interpretation for Eqs. (26) and (28). As was shown earlier, by writing $M(t+d t)=e^{\xi \sigma_{3}} e^{-2 \eta \vec{\sigma} \cdot \hat{n}_{2 \theta}} e^{\xi \sigma_{3}}$ with $\xi=\chi d t$, Eq. (9) can be regarded as a velocity addition problem $(\delta \vec{v}+\vec{v}+\delta \vec{v})$ with $\vec{v}=(\tanh 2 \eta) \hat{n}_{2 \theta}$ and $\delta \vec{v}=-\chi d t \hat{n}_{3}$. Here $\hat{n}_{2 \theta}=c_{2 \theta} \hat{n}_{3}+s_{2 \theta} \hat{n}_{1}$. The resultant Lorentz boost has the rapidity $2(\eta+d \eta)$, with $d \eta=\delta v_{\|}=-\chi d t c_{2 \theta}$, while its angle with respect to the third axis is increased by $2 d \theta=2 \delta v_{\perp} /|\vec{v}|=2 \chi d t s_{2 \theta} / \tanh 2 \eta$.

\section{Phase Portraits}

To better understand the qualitative effects of the RGE, we rewrite Eqs. (26-28) in terms of a slightly different combination of the physical mass eigenvalues [4]

$$
z=\operatorname{coth} 2 \bar{\eta}=\frac{m_{2} e^{2 i \phi}+m_{1} e^{-2 i \phi}}{m_{2} e^{2 i \phi}-m_{1} e^{-2 i \phi}}
$$


In terms of this variable, the RGEs are

$$
\begin{aligned}
\frac{d z}{d t} & =4 \chi c_{2 \theta} \frac{\left(-1+z^{2}\right) z^{*}}{z+z^{*}} \\
\frac{d \theta}{d t} & =2 \chi s_{2 \theta} \frac{|z|^{2}}{z+z^{*}}
\end{aligned}
$$

where $z^{*}$ is the complex conjugate of $z$.

The neutrino mass matrix, Eq. (13), possesses various symmetries. For example, under the transformation

$$
\theta \rightarrow \frac{\pi}{2}-\theta
$$

accompanied by an interchange of the mass eigenvalues

$$
\begin{aligned}
& \eta \rightarrow-\eta \quad\left(m_{2} \leftrightarrow m_{1}\right) \\
& \phi \rightarrow-\phi
\end{aligned}
$$

or, equivalently,

$$
z \rightarrow-z
$$

the diagonal elements are invariant while the off-diagonal elements change sign. However the sign of the off-diagonal mass-matrix element, which is the RGE invariant (Eq. (14)), is not a physical observable since it may be absorbed in the unphysical phases with a redefinition of the neutrino wave function $\left(\nu_{\mu}, \nu_{\tau}\right) \rightarrow\left(\nu_{\mu},-\nu_{\tau}\right)$. Thus the mass matrix and the RGE evolution equations respect this symmetry.

The evolution equations in terms of complex $z$ and $\theta$, Eqs. (31) and (32), have five fixed points. The evolution is always towards or away from one of these fixed points, i.e. the parameters do not evolve towards infinity. Of the five fixed points, only three are physically distinct in that the above mass matrix symmetry maps two fixed points into two others. We have obtained the stability of the fixed points for t decreasing (i.e. running towards the infrared) in the SM by finding the eigenvalues of the Jacobian (see e.g. [6]). The attractive fixed points are $\theta=0, z=+1$ and $\theta=\pi / 2, z=-1$ which corresponds to no mixing and a massless muon-neutrino. The repulsive fixed points are $\theta=0, z=-1$ and $\theta=\pi / 2, z=+1$ which corresponds to no mixing and a massless tau-neutrino. The final fixed point is a saddle point, attractive in some directions and repulsive in others, and it is at $\theta=\pi / 4, z=0$. This point corresponds to maximal mixing with equal magnitude but opposite sign mass eigenvalues. These stabilities are for the SM, for the MSSM all evolution directions are reversed, so the attractors and repulsors are reversed also.

These RGEs and the fixed points are graphically displayed in Figs. 여 where the direction fields are plotted for t decreasing. Starting from some initial point specified by the high energy theory, the evolution of $z$ and $\theta$ follow a trajectory in these spaces and the plotted unit arrows show the directions tangent to this trajectory. The direction field is independent of $\mathrm{t}$ and $\chi$. The trajectories can be found by taking the RGE equations, Eqs. (31) and (32), dividing one by the other and integrating to get

$$
\frac{\sin ^{2} 2 \theta}{z^{2}-1}=\zeta
$$


where $\zeta$ is a constant. This equation is just the square of the RGE invariant, Eq. (14).

If the mass matrix is initially real, then RGE evolution preserves this. The evolution of real parameters, $\operatorname{Im}(z)=0$, is shown in Fig. 1. The symmetries of the neutrino mass matrix, Eqs. (33) and(36), are apparent. The dark curves in Fig. 11 correspond to $\zeta=-1$ in Eq. (37). These curves connect all of the fixed points. Note that half of these dark curves flow towards the maximal mixing fixed point and end there, the only trajectory that does so. Trajectories with $\zeta<-1$ or $\zeta \geq 0$ are those on the left and right of the plot and they can pass through maximal mixing. Trajectories with $-1<\zeta<0$ connect positive and negative $z$ values and never attain maximal mixing.

The general behavior for $\operatorname{Im}(z) \neq 0$ is shown in Fig. 2, where the vector field is plotted in the full, 3-D parameter space. This figure is for the range $0<\operatorname{Re}(z), 0<\operatorname{Im}(z)$, $0<\theta<\pi / 2$, so the 'floor' of this figure corresponds to the right half of Fig. 1. The behavior in other regions of the parameter space can be obtained from this by symmetry operations. The three fixed points are shown using a dark sphere, light sphere and a cuboid to denote the attractor, repellor and saddle point. Note that the figure resembles the vector field of a dipole, with evolution away from the repellor and towards the attractor.

The evolution equations are singular at $\operatorname{Re}(z)=0$ if $\operatorname{Im}(z) \neq 0$. Thus large changes in the mixing angle are possible then, despite the small size of $\chi$. The behavior in this region is shown in Fig. 3. Here the direction field is plotted for the $\operatorname{Im}(z), \operatorname{Re}(z)$ plane. Note that this direction field is independent of the magnitude of $\theta$, except that the direction of flow does depend on the sign of $\cos 2 \theta$. The fixed points for $\theta=0$ and $\theta=\pi / 4$ are included in the plot. As Fig. 3 shows, the flow across the $\operatorname{Re}(z)=0$ value does not occur if $\operatorname{Im}(z) \neq 0$. The flow on the right-hand side is towards the stable fixed point there. On the left-hand side, the flow is away from the repulsor, but at the same time the mixing angle is increasing and eventually exceeds $\theta=\pi / 4$ at which point the flow direction reverses, the repulsor becomes an attractor and the evolution approaches the now stable fixed point by retracing its path in the $\operatorname{Im}(z), \operatorname{Re}(z)$ plane.

\section{$5 \quad$ Analysis}

We turn now to a quantitative analysis of our results. First, we consider the case of real mass matrices, $\phi_{0}=0$ or $\pi / 4$, so that $\Delta \alpha=0$ from Eq. (16) and

$$
\tan 2 \theta=\frac{s_{2 \theta_{0}} / \operatorname{ch} 2 \xi}{c_{2 \theta_{0}}-\Sigma_{R} \tanh 2 \xi}
$$

where, from Eq. (17)

$$
\Sigma_{R}= \begin{cases}\left(\frac{m_{2}+m_{1}}{m_{2}-m_{1}}\right)_{0}=\operatorname{coth} 2 \eta_{0}, & \phi_{0}=0 \\ \left(\frac{m_{2}-m_{1}}{m_{2}+m_{1}}\right)_{0}=\tanh 2 \eta_{0}, & \phi_{0}=\pi / 4 .\end{cases}
$$

Since $|\xi| \ll 1,\left(|\xi| \sim 10^{-5}\right.$ for the SM and $|\xi| \leq \mathcal{O}\left(10^{-2}\right)$ for the MSSM), we see immediately that for $\phi_{0}=\pi / 4$ (opposite sign mass eigenvalues), $\theta \simeq \theta_{0}$. For $\phi_{0}=0$ (same sign 
masses), $\tan 2 \theta$ exhibits the resonance behavior, and maximal mixing is obtained if

$$
c_{2 \theta_{0}}=\tanh 2 \xi / \tanh 2 \eta_{0}, \quad\left(\phi_{0}=0\right) .
$$

For a generic value of $\theta_{0}<\pi / 4$, this condition can be fulfilled provided that $\xi$ and $\eta_{0}$ are of the same sign, and that $\eta_{0}$ is very small (nearly degenerate masses). In terms of the masses, if we define

$$
\delta_{0}=1-\left(m_{1} / m_{2}\right)_{0}
$$

then the resonance condition becomes

$$
c_{2 \theta_{0}} \simeq 4 \xi / \delta_{0} \quad\left(\phi_{0}=0\right) .
$$

For SM, $\xi<0$, it is seen that $\theta<\theta_{0}$, if $m_{2}>m_{1}$. That is, under "normal" situations, RGE drives the mixing angle toward zero as the energy decreases. For MSSM, $\xi>0$, we see that $\theta$ can become large if there is near degeneracy, $m_{2} \simeq m_{1}\left(m_{2}>m_{1}\right)$. Of course, if $\theta_{0}>\pi / 4$ (which amounts to the interchange $m_{1} \leftrightarrow m_{2}$ ), or if $m_{1}>m_{2}$, the direction of the running of the mixing angle is reversed. These conclusions agree with earlier results obtained by numerical integrations of the RGEF.

At the same time, the RGE invariant (Eq. (14)) gives the running of the mass ratio

$$
\begin{array}{ll}
s_{2 \theta} \operatorname{sh} 2 \eta=s_{2 \theta_{0}} \operatorname{sh} 2 \eta_{0}, & \left(\phi_{0}=0\right) \\
s_{2 \theta} \operatorname{ch} 2 \eta=s_{2 \theta_{0}} \operatorname{ch} 2 \eta_{0}, & \left(\phi_{0}=\pi / 4\right) .
\end{array}
$$

While $\eta \sim \eta_{0}$ for $\phi_{0}=\pi / 4$, for $\phi_{0}=0$ and at the resonance, $s_{2 \theta} \gg s_{2 \theta_{0}}$, it is seen that $\operatorname{sh} 2 \eta \ll \operatorname{sh} 2 \eta_{0}$, i.e., the masses are driven toward even more degeneracy as $\theta$ becomes maximal from a small $\theta_{0}$.

For complex mass matrices $\left(\phi_{0} \neq 0\right.$ or $\left.\pi / 4\right)$, we need to use Eq. (15). From Eq. (28), it is seen that for $\chi \ll 1, d \theta / d t$ is appreciable only if $\eta \ll 1$. In other words, RGE effect can be large only if $\delta=1-\left(m_{1} / m_{2}\right) \ll 1$, as for real matrices. We will confine our discussion in this parameter region $\delta \ll 1$. Further, for definiteness, we assume that $\xi>0$ and $\eta_{0}>0$. (The other possibilities can be easily analyzed.) For finite values of $\phi_{0}$, it is easy to see that

$$
\begin{aligned}
\Sigma_{R} & \simeq \delta_{0} /\left(2 s_{2 \phi_{0}}^{2}\right) \\
\Sigma_{I} & \simeq-\cot 2 \phi_{0}
\end{aligned}
$$

where higher order terms in $\delta_{0}$ are neglected. The condition for maximal mixing is then

$$
\frac{\delta_{0}}{4 \xi} \simeq\left(c_{2 \theta_{0}} s_{2 \phi_{0}}^{2}+c_{2 \phi_{0}}^{2} / c_{2 \theta_{0}}\right), \quad\left(\phi_{0} \neq 0, \pi / 4\right)
$$

This reduces to Eq. (42) as $\phi_{0} \rightarrow 0$. However, when $\phi_{0}=\pi / 4$, we had found earlier that there is no resonance. This means that the limit $\phi_{0} \rightarrow \pi / 4$ is actually very delicate.

\footnotetext{
${ }^{2}$ Note also that, for general values of $\phi$, similar behaviors can be deduced from Eq. (28)
} 
As we will see, what happens is that the width of the resonance also shrinks to zero as $\phi_{0} \rightarrow \pi / 4$. This behavior is borne out in the numerical calculation shown in Figs. 1 and 5.

Although the analytic formulae for $\theta, \eta$ and $\phi$ have been explicitly given, as we have seen in the previous analyses, their detailed behaviors are quite intriguing. It is useful to have an overview of these functions. For this purpose we present, in Figs. 1 and 5 , numerical calculations of the function $\theta(t)$. Traditionally the plot of $\theta(t)$ is made with $t$ as the variable. However, as shown in Eqs. (42) and (47), the interesting region is determined by $\delta_{0} / \xi \sim \mathcal{O}(1)$. So an equivalent way is to plot $\theta$ vs. $\delta_{0}\left(\right.$ or $\left.\left(m_{1} / m_{2}\right)_{0}\right)$, while keeping $t$ as a constant parameter. In Fig. 4 , we present a 3 D plot of $\sin ^{2} 2 \theta$ vs. $\left(m_{1} / m_{2}\right)_{0}$ and $4 \phi_{0}$. We take a positive $\xi=\chi t=10^{-2}$ (corresponding to MSSM with $\tan \beta=40$ and $\left.t=\frac{1}{16 \pi^{2}} \ln \left(m_{Z} / M_{X}\right), M_{X} \simeq 3 \times 10^{10} \mathrm{GeV}\right)$. This sets the scale in $\left(m_{1} / m_{2}\right)_{0}$, with $\left(1-m_{1} / m_{2}\right)_{0}=\delta_{0} \sim 10^{-2}$. Thus for a given initial value $\theta_{0}=\pi / 12$, the plot gives $\sin ^{2} 2 \theta$ as a function of the initial parameters $\left(m_{1} / m_{2}\right)_{0}$ and its phase $4 \phi_{0}$. The resonant behavior of $\sin ^{2} 2 \theta$ is obvious. The position of the maximum is, for $\phi_{0}=0$, at $\delta_{0} \simeq 8 \xi / \sqrt{3}$. The position of the maximum shifts with changing $\phi_{0}$, and is well described by Eq. (47).

Fig. 5 shows $2 \mathrm{D}$ slices of the $3 \mathrm{D}$ plot in Fig. 6 . We plot, for various values of $\phi_{0}$, $\sin ^{2} 2 \theta$ vs. $\left(m_{1} / m_{2}\right)_{0}$. It shows clearly the shifting positions of the maxima as $\phi_{0}$ changes. Also notice the change of width as a function of $\phi_{0}$. It can be shown that, provided $4 \xi^{2} \ll c_{2 \theta_{0}}^{2} \tan ^{2} 2 \phi_{0}$, the width is given by

$$
\Delta\left(m_{1} / m_{2}\right)_{0} \simeq 8 \xi c_{2 \phi_{0}}\left(s_{2 \theta_{0}} / c_{2 \theta_{0}}^{2}\right) \sqrt{c_{2 \phi_{0}}^{2}+c_{2 \theta_{0}}^{2} s_{2 \phi_{0}}^{2}}
$$

so that the resonance disappears as $\phi_{0} \rightarrow \pi / 4$, in agreement with Eqs. (38) and (39). Finally, the singular nature of the limit $\phi_{0} \rightarrow \pi / 4$ is confirmed when we compare the curves with $\phi_{0}=0.9 \pi / 4$ and $\phi_{0}=\pi / 4$.

We do not show 3D plots for $\eta$ and $\phi$. Since from the RGE invariant,

$$
\begin{aligned}
& s_{2 \theta} \operatorname{sh} 2 \eta c_{2 \phi}=s_{2 \theta_{0}} \operatorname{sh} 2 \eta_{0} c_{2 \phi_{0}}, \\
& s_{2 \theta} \operatorname{ch} 2 \eta s_{2 \phi}=s_{2 \theta_{0}} \operatorname{ch} 2 \eta_{0} s_{2 \phi_{0}},
\end{aligned}
$$

once we know $\theta$, they can be easily determined. Note that for nearly degenerate masses $(\eta \ll 1)$, it can be seen from Eq. (50) that there is an anti-correlation between $\theta$ and $\phi$ with the phase $\phi$ being minimal at maximal mixing. Also, at maximal mixing and with nearly degenerate masses, simple expressions for the neutrino mass ratio and the relative phase can be deduced from Eqs. (49) and (50),

$$
\begin{aligned}
s_{2 \phi} & \simeq s_{2 \theta_{0}} s_{2 \phi_{0}} \\
\delta & \simeq \delta_{0} s_{2 \theta_{0}} c_{2 \phi_{0}} / \sqrt{1-s_{2 \theta_{0}}^{2} s_{2 \phi_{0}}^{2}}
\end{aligned}
$$

where $\delta=1-m_{1} / m_{2}$. As can be seen from Eq. (52), for small $\theta_{0}$, the neutrino masses become more degenerate at maximal mixing. 


\section{Conclusions}

We have examined the RGEs for the physical, two-flavor, neutrino parameters: mixing angle, mass ratio and their relative phase in the SM and MSSM. These equations turn out to have relatively simple forms which we analyze in detail. The qualitative nature of the evolution is clearly illustrated in our vector field plots. A more quantitative description is also given using our exact, analytical solution for the evolution. In addition, we have also found a complex RGE invariant which correlates the running of the three physical parameters.

The phase portraits show the direction of the evolution throughout the parameter space. The fixed points and their stability are included on the plots. It should be noted that maximal mixing $(\theta=\pi / 4)$ is a saddle point in both the SM and MSSM, and the infrared stable point (attractor) in the SM (MSSM) corresponds to no mixing and a massless muon-neutrino (tau-neutrino). Thus to get maximal mixing at the experimental scale requires a particular choice of mass ratio and phase at the high-energy scale.

The parameter choices that give maximal mixing have been calculated with our exact solution. As is well-known for real parameters, large evolution requires nearly degenerate masses. With the addition of a complex phase, the peak position shifts and the resonance region becomes narrower, so to achieve maximal mixing at the experimental scale requires a finer tuning of parameters at the high-energy scale.

In the literature, considerable interest has been focused on the possibility of generating large mixing through RGE running. The detailed solution for RGE suggests both opportunities and limitations for this scenario. Since the end result depends very sensitively on the initial conditions, any such model must be treated carefully. In particular, one needs to study the three flavor problem in detail. Although it is very difficult to obtain exact solutions in this case, approximate solutions seem well within reach. We hope to return to this problem in the future.

Acknowledgements: T. K. K. and G.-H. W. are supported in part by DOE grant No. DE-FG02-91ER40681 and No. DE-FG03-96ER40969, respectively. J.P. is supported by NSF grant PHY-0070527.

\section{References}

[1] Super Kamiokande Collaboration, Y. Fukuda et al., Phys. Rev. Lett. 82 (1999) 2644; S. Hatakeyama et al., Kamiokande Collaboration, Phys. Rev. Lett. 81 (1998) 2016.

[2] B.T. Cleveland et al., Astrophys. J 496 (1998) 505; W. Hampel et al., GALLEX Collaboration, Phys. Lett. B388 (1996) 384; D.N. Abdurashitov et al., SAGE Collaboration, Phys. Rev. Lett. 77 (1996) 4708; K.S. Hirate et al., Kamiokande Collaboration, Phys. Rev. Lett. 77 (1996) 1683. 
[3] K. Babu, C. N. Leung, and J. Pantaleone, Phys. Lett. B319, 191 (1993), hepph/9309223; P. H. Chankowski and Z. Pluciennik, Phys. Lett. B316, 312 (1993), hep-ph/9306333.

[4] J.A. Casas, J.R. Espinosa, A. Ibarra, and I. Navarro, Nucl. Phys. B573, 652 (2000), hep-ph/9910420; ibid, B569, 82 (2000), hep-ph/9905381.

[5] J. Ellis and S. Lola, Phys. Lett. B458, 310 (1999), hep-ph/9904279; P.H. Chankowski, W. Krolikowski, and S. Pokorski, Phys. Lett. B473, 109 (2000), hep-ph/9910231; N. Haba et. al., Prog. Theor. Phys. 103, 145 (2000), hep-ph/9908429; N. Haba and N. Okamura, Eur. Phys. J. C14, 347 (2000), hep-ph/9906481; N. Haba, Y. Matsui, and N. Okamura, Prog. Theor. Phys. 103, 807 (2000), hep-ph/9911481; M. Tanimoto, Phys. Lett. B360, 41 (1995), hep-ph/9508247; S.F. King and N. Nimai Singh, Nucl. Phys. B591, 3 (2000), hep-ph/0006229; Z.-Z. Xing, Phys. Rev. D63, 057301 (2001), hep-ph/0011217; K.R.S. Balaji, R.N. Mohapatra, M.K. Parida, and E.A. Paschos, hep-ph/0011263.

[6] S. Strogatz, Nonlinear Dynamics and Chaos (Addison-Wesley, New York, 1994).

[7] M. Gell-Mann, P. Ramond and R. Slansky, in Supergravity, eds. P. van Nieuwenhuizen and D. Freedman (North Holland, Amsterdam, 1979); T. Yanagida, in Proceedings of the Workshop on Unified Theory and Baryon Number in the Universe, eds. O. Sawada and A. Sugamoto (KEK, 1979).

[8] T. K. Kuo, G.-H. Wu, and S.-H. Chiu, Phys. Rev. D 62, 051301 (2000), hepph/0003066.

[9] T.K. Kuo, S.-H. Chiu, G.-H. Wu, hep-ph/0011058, Eur. Phys. J. C, in print. 


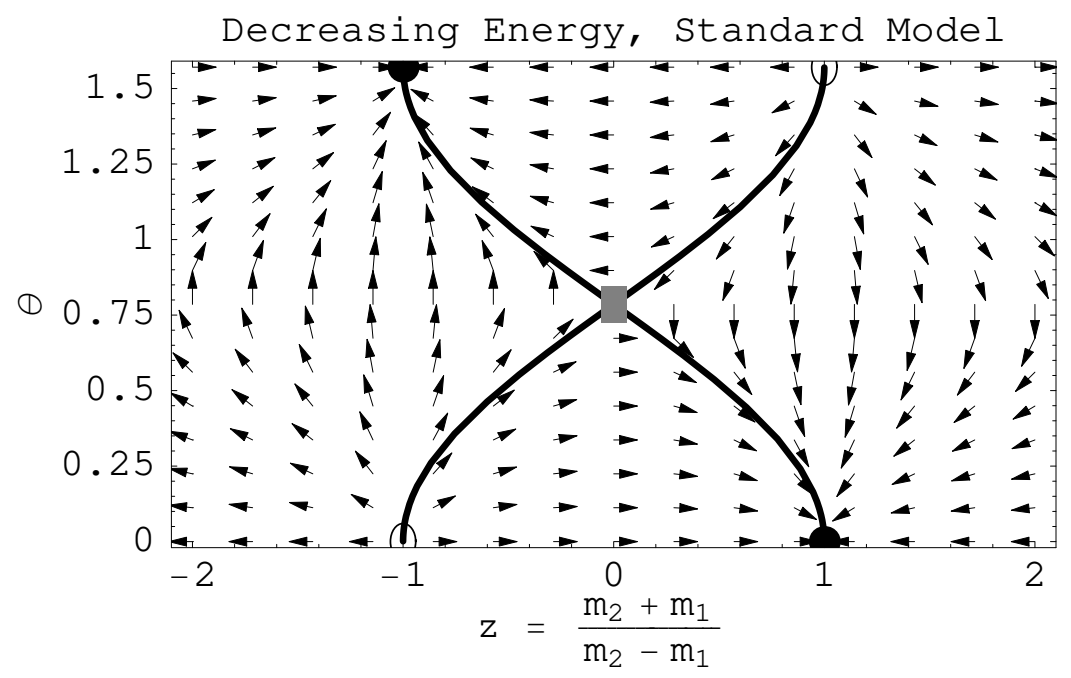

Figure 1: Phase portrait for the Standard Model RG equations when $\operatorname{Im}(z)=0$. The different fixed points are shown with solid circles, open circles and grey square denoting attractors, repellors and saddle point. The solid curve shows the trajectories that connect the fixed points. The arrows are reversed for the MSSM. 


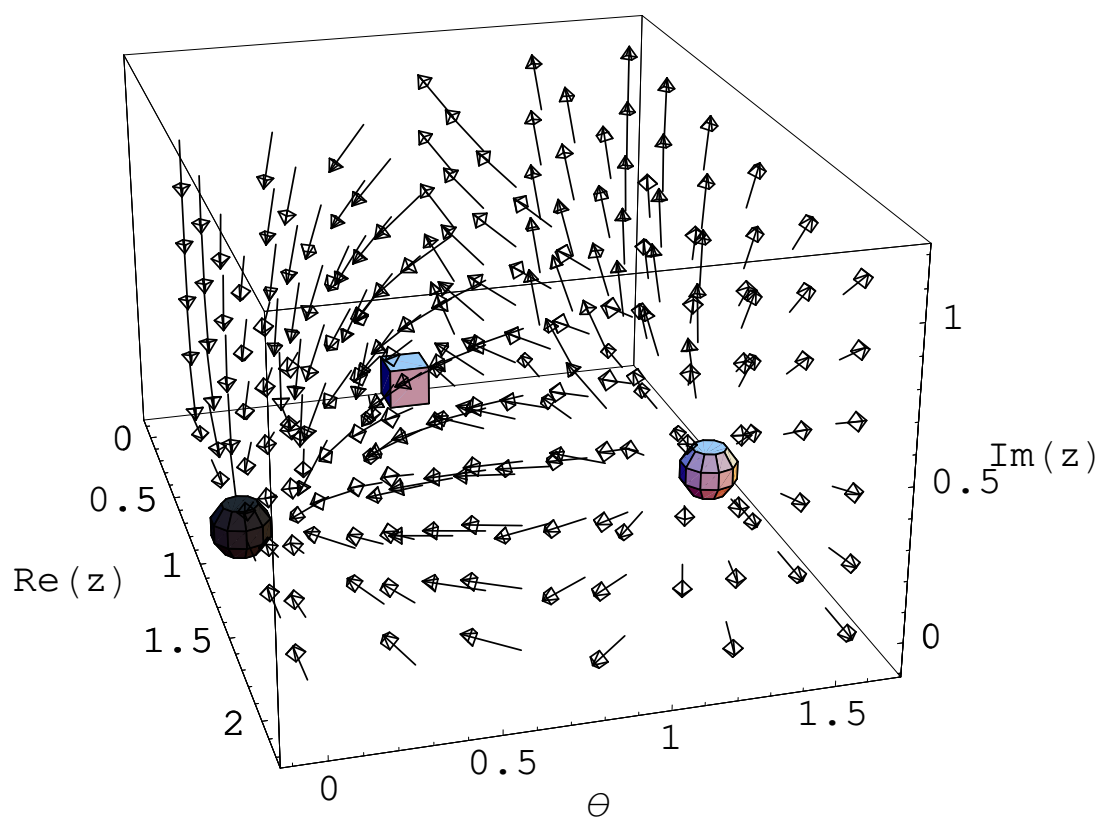

Figure 2: The SM RGE evolution in $\operatorname{Re}(z), \theta, \operatorname{Im}(z)$ space, for the range $0<\operatorname{Re}(z)$, $0<\operatorname{Im}(z), 0<\theta<\pi / 2$. The different fixed points are all confined to the $\operatorname{Im}(z)=0$ plane. They are shown using a dark sphere, light sphere and a cuboid which denote the attractor, repellor and saddle point. 


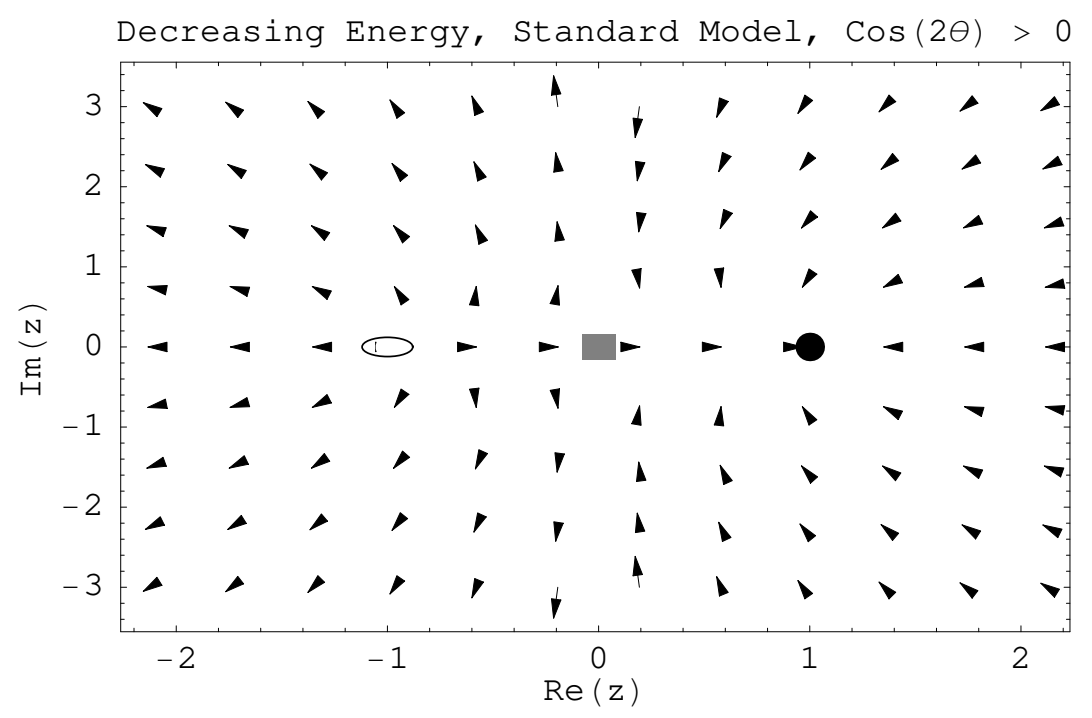

Figure 3: The SM RGE evolution in the $\operatorname{Re}(z), \operatorname{Im}(z)$ plane for $\cos 2 \theta>0$. The $\theta=0$ fixed points are shown with solid circle, open circle and grey square denoting attractor, repellor and saddle point. For $\cos 2 \theta<0$, the figure is similar except all directions (and stabilities) are reversed. Note that $\theta$ also evolves. 


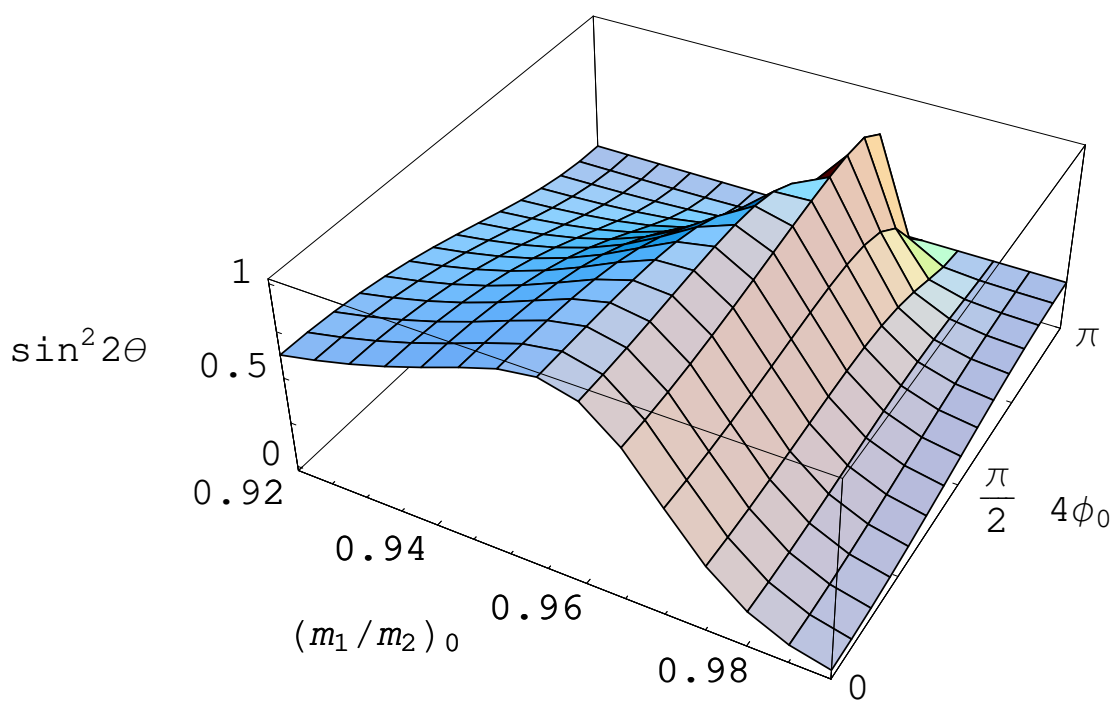

Figure 4: $3 \mathrm{D}$ plot for $\sin ^{2} 2 \theta$ vs. initial values $\left(m_{1} / m_{2}\right)_{0}$ and $4 \phi_{0}$. The inputs are fixed at $\theta_{0}=\pi / 12$ and the RGE factor $\xi=0.01$ (MSSM). 


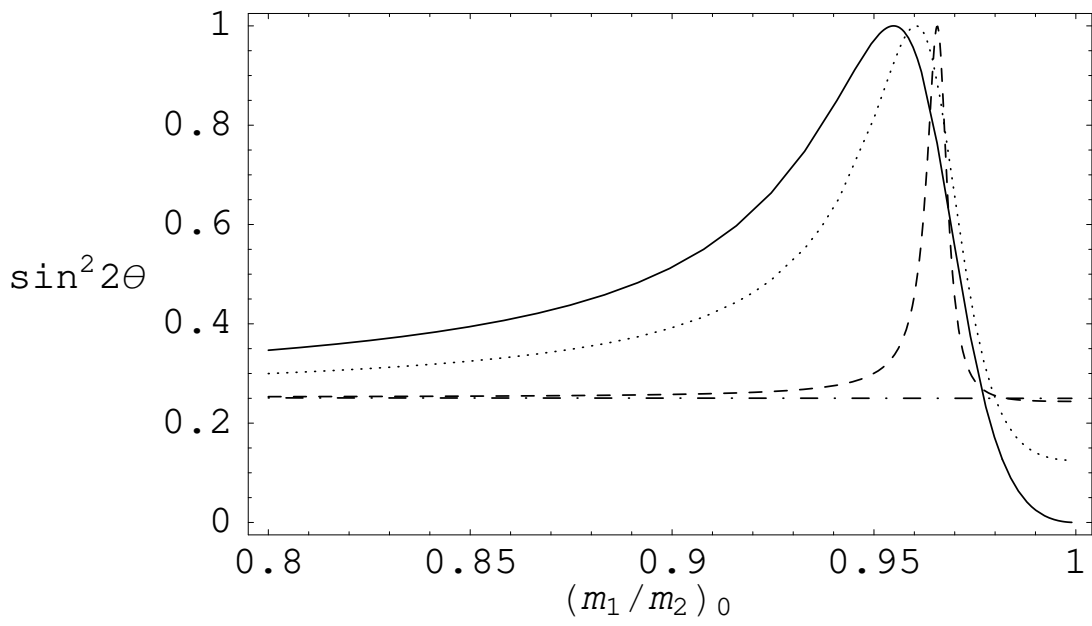

Figure 5: $\sin ^{2} 2 \theta$ vs. $\left(m_{1} / m_{2}\right)_{0}$ with inputs $\theta_{0}=\pi / 12$ and $\xi=0.01$ (MSSM). The solid, dotted, dashed, and dot-dashed curves correspond to $4 \phi_{0}=0, \pi / 2,0.9 \pi$ and $\pi$ respectively. 